\title{
Review of existing and development of a novel mathematical model for supercritical $\mathrm{CO}_{2}$ cycles working fluid
}

\author{
Marcin Wołowicz ${ }^{*}$ Jarosław Milewski, and Piotr Lis \\ Institute of Heat Engineering, Warsaw University of Technology, Nowowiejska 21 Street 00-665 Warsaw, Poland
}

\begin{abstract}
The paper aims to compare the models of working fluids against experimental data for carbon dioxide close to its critical conditions. Fortunately, most of the work is already done and published where the authors compared the models based on the equation of the state (EoS). There are a few other models which were not investigated, thus we would like to add a few new results here and focus only on nearcritical properties where the biggest deviation between experimental and calculated properties can be observed. The area of interest was pressure range of $7.39-20 \mathrm{MPa}$ and temperature range of 304-340 K just above fluid critical point (7.39 MPa, 304.25 K). Model validation was performed for density and heat capacity as one of the most important parameters in preliminary cycle analysis.
\end{abstract}

\section{Introduction}

The paper aims to compare the models of working fluids against experimental data for carbon dioxide close to its critical conditions. Fortunately, most of the work is already done and published by Zhao et al [1], where the authors compared the models based on the equation of the state $(\mathrm{EoS})$ as follows:

1. Peng Robinson (PR)

2. Peng-Robinson combined with the BostonMathias alpha function (PR-BM)

3. Soave-Redlich-Kwong (SRK)

4. Benedict-Webb-Rubin modified by Starling and Nushiumi (BWRS)

5. Lee-Kesler-Plöcker (LKP)

6. Span-Wagner (SW)

They concluded that in terms of pure-component behavior, among the six studied EoS, the Span-Wagner EoS is the one that leads to the most accurate predictions of the $\mathrm{CO}_{2}$ in the near-critical and supercritical regions. In terms of cycle efficiency, it has been observed that the influence of the EoS choice is limited but not necessarily negligible. However, from a component-sizing point of view, they found that certain deviations can be considered as high, leading to economic issues due to over or underestimation of equipment costs (at level of $10 \%$ ). Thus, the SW EoS is recommended for evaluating the performances of a SC- $\mathrm{CO}_{2}$ Brayton cycle in order to avoid inaccurate predictions in terms of equipment sizing and optimization.

There are a few other models which were not investigated in [1], thus we would like to add a few new results here and focus only on near-critical properties where the biggest deviation between experimental and calculated properties can be observed. The area of interest was pressure range of $7.39-20 \mathrm{MPa}$ and temperature range of $304-340 \mathrm{~K}$ just above fluid critical point (7.39 MPa, $304.25 \mathrm{~K}$ ). Model validation was performed for density and heat capacity as one of the most important parameters in preliminary cycle analysis.

The following fluid models not included in Zo study were considered for modeling super critical $\mathrm{CO}_{2}$ cycles focusing on near-critical point area:

1. Klimeck (GERG2008)

2. Cubic-Plus-Association (CPA)

3. Benedict-Webb-Rubin-Lee-Starling (BWR-LS)

4. Peng-Robinson-Wong-Sandler (PRWS)

5. Predictive Redlich-Kwong-Soave (PRKS)

6. Redlich-Kwong-Soave (RKS) with BostonMathias alpha function (RKS-BM)

7. Redlich-Kwong-Soave-Wong-Sandler (RKSWS)

8. Schwarzentruber-Renon (SR-POLAR)

The Span-Wagner EoS was used as a reference as it was proofed to be the most accurate in previous studies. Investigation was done using Aspen One ${ }^{\circledR}$ simulation software.

\section{Supercritical cycles}

Supercriticality is a hybrid state, where distinct liquid and gas phases do not exist. A supercritical fluid is dense, like a liquid, but expands to fill a volume the way a gas does. Small changes in temperature near the critical point-about $31^{\circ} \mathrm{C}$ cause big changes in density, similar to evaporating where the liquid changes to a vapor. The

* Corresponding author: marcin.wolowicz@itc.pw.edu.pl 
density change, however, is more than two orders of magnitude smaller that the density change when, e.g., water becomes steam at atmospheric pressure. Similarly, it takes a lot of energy to increase the temperature a small amount when the fluid is near the critical point, much the way the heat of vaporization requires great amount of energy. Consequently, a large spike in heat capacity occurs near the critical point of $\mathrm{CO}_{2}$.

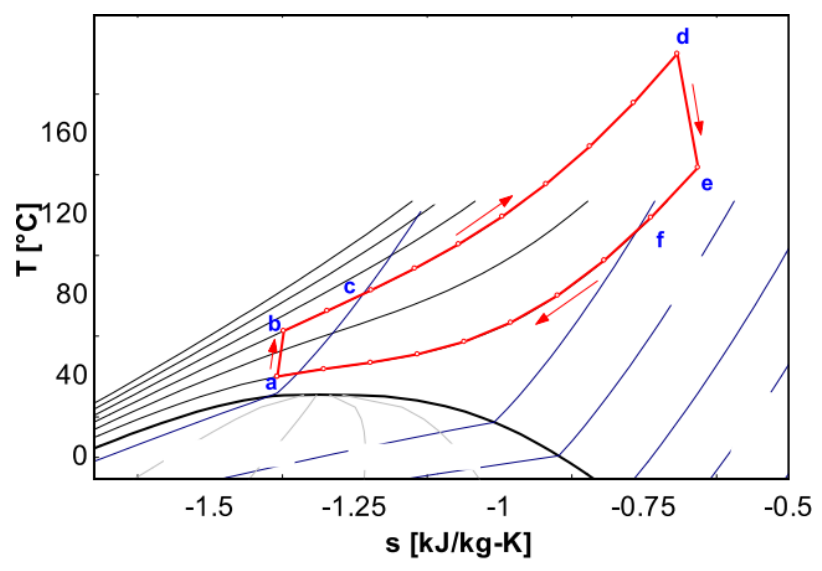

Fig. 1. Carbon dioxide Brayton cycle T-S chart [2].

Both supercritical and transcritical cycles are presented in Fig. 1 and Fig. 2 respectively. It can be seen from T-S chart, that the plot of the working fluid of a supercritical cycle is located in the supercritical area throughout all the points of the cycle. In case of a transcritical cycle a part of the plot is below the critical point, nonetheless most of the points are situated over the saturation line, therefore both cycles are usually related to supercritical cycles in the literature.

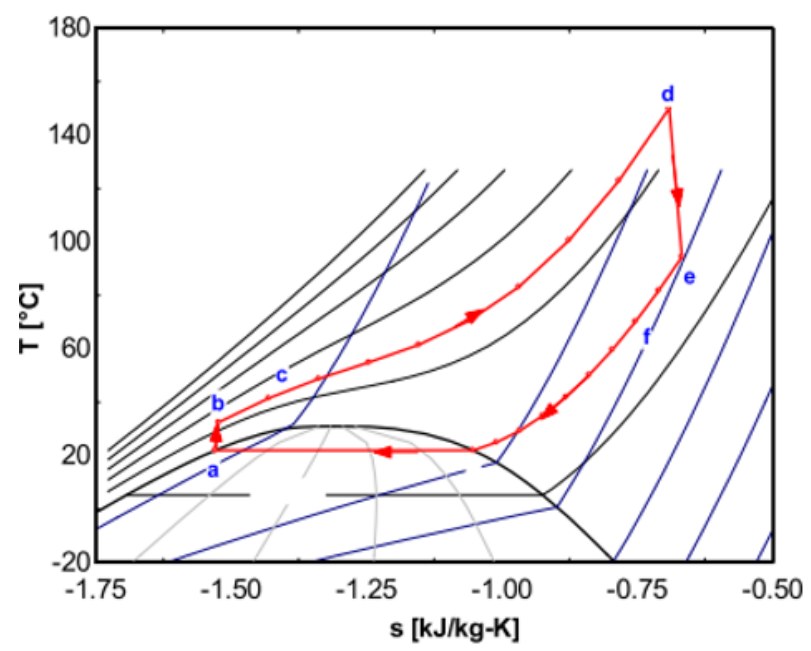

Fig. 2. Carbon dioxide transcritical cycle T-S chart [2].

Both cycles consists of four processes, namely: compression (pumping) (a-b), isobaric heat supply (b-d), expansion (d-e) and isobaric heat rejection (e-a). It is worth to note that the transcritical cycle has considerably lower low cycle temperature.
Since carbon dioxide used as a working fluid in both supercritical and transcritical power cycles operates mostly in the supercritical area, the ideal gas model cannot be applied to obtain the working parameters and the cycle performance. On the contrary, carbon cycle behavior deviates substantially from an ideal gas behavior particularly near the critical point. Therefore, the best suited real gas equation of state has to be selected to calculate $\mathrm{CO}_{2}$ parameters.

Obviously, all the equations of state designed to describe a real gas behavior that have been developed in the past are burdened with error. However, there are some of them that are usually recommended in the literature. $\mathrm{Oh}$ et al. [4] proposed the Lee-Kesler-Plocker equation of state as the most accurate and used it for all computer calculations. A comparison made by Mazzoccoli in 2012 [4] proved that out of 17 examined equations of state, the Span-Wagner [5] equation has the lowest error in the density prediction in every region analyzed. Wang et al. [6] performed an analysis which aimed at developing an improved density correlation for supercritical $\mathrm{CO}_{2}$. An experimental apparatus was designed to measure accurately the density of supercritical $\mathrm{CO}_{2}$ in temperature range from 303 to $473 \mathrm{~K}$, the pressure from 3 to $60 \mathrm{MPa}$.

Then, the relative error between the experimental and calculated density values for a number of frequently used equation of state was evaluated. The high-precision equations, such as the MBWR, Lee-Kesler, SpanWagner, and Sun-Kiselev-Ely equations of state, proved to provide the most accurate values. However, the maximum relative error can reach approximately $20 \%$ in the near critical region, thus the authors proposed an error correction term to the original equations. The corrected Lee-Kesler, MBWR, Span-Wagner, and SunKiselev-Ely equations can precisely predict the density of supercritical $\mathrm{CO}_{2}$, and the average relative error can be restricted to within $1.5 \%$. Besides, the new polynomial density calculation formula with 28 parameters was proposed based on the experimental data. This formula can restrict the average relative error of density to $2.0 \%$ across the whole studied range.

\section{Theory}

Selected EoS can be divided into three categories similar as in paper [1]:

1. Helmoholtz-type: GERG2004

2. Virial-type: BWR-LS

3. Cubic-type: PRWS, PRKS, CPA, RKS-BM, RKSWS, SR-POLAR

\subsection{Klimeck (GERG2004)}

The GERG2004 equation of state is based on a multifluid approximation explicit in the reduced Helmholtz free energy. This model was developed under the auspices of the Groupe Européen de Recherches 
Gazières (GERG) in cooperation with the German technical and Scientific Association on Gas and Water and European natural gas companies (E.ON Ruhrgas, Germany; Enagas, Spain; Gasunie, The Netherlands; Gaz de France, France; Snam Rete Gas, Italy; and Statoil, Norway). The model of carbon dioxide is based on work done by Klimeck [14] and is valid for temperature range of $216-900 \mathrm{~K}$ and pressure up to 300 $\mathrm{MPa}$. EoS contains 22 terms. As it is based on SW EoS the prediction accuracy is expected to be similar.

\subsection{Benedict-Webb-Rubin-Lee-Starling (BWR- LS)}

The Benedict-Webb-Rubin-Lee-Starling EoS [15] is a generalization by Lee and Starling of the Virial EoS for pure fluids by Benedict, Webb and Rubin. The equation is used for non-polar components, and can manage hydrogen-containing systems.

\subsection{Peng-Robinson-Wong-Sandler (PRWS)}

This model uses the Peng-Robinson equation-of-state for pure compounds. The mixing rules are the predictive Wong-Sandler rules. The binary parameter PRWSK is used for the kit. Several alpha functions can be used in the Peng-Robinson-Wong-Sandler equation-of-state model for a more accurate description of the pure component behavior. The pure component behavior and parameter requirements are described in Peng-Robinson, and in Peng-Robinson Alpha Functions.

\subsection{Predictive Redlich-Kwong-Soave (PRKS)}

This model uses the Redlich-Kwong-Soave equation-ofstate for pure compounds. The mixing rules are the predictive Holderbaum rules, or PSRK method. Several alpha functions can be used in the PSRK equation-ofstate model for a more accurate description of the pure component behavior. The pure component behavior and parameter requirements are described in RedlichKwong-Soaveand in Soave Alpha Functions.

\subsection{Cubic-Plus-Association (CPA)}

The CPA method represents the Cubic-Plus-Association EOS model developed by Kontogeorgis and co-workers (Kontogeorgis, Voutsas, Yakoumis, Tassios, IECR 1996). The model combines the SRK cubic EOS with an association term similar to that of SAFT, as present in the PC-SAFT model. Mixing rules apply to the cubic, whereas combining rules are used for the association term. The model's applicability covers the VLE and VLLE of mixtures containing hydrocarbons and polar/associating chemicals such as water, alcohols, glycols, esters, and organic acids.

\subsection{Redlich-Kwong-Soave (RKS) cubic equation of state with Boston-Mathias alpha function (RKS-BM)}

The RKS-BM EoS is the Redlich-Kwong-Soave equation-of-state with the Boston-Mathias alpha function. It is recommended for hydrocarbon processing applications, such as gas-processing, refinery, and petrochemical processes. Its results are comparable to those of the Peng-Robinson-Boston-Mathias equationof-state.

\subsection{Redlich-Kwong-Soave-Wong-Sandler (RKSWS)}

This EoS uses the Redlich-Kwong-Soave equation-ofstate for pure compounds. The predictive Wong-Sandler mixing rules are used. Several alpha functions can be used in the Redlich-Kwong-Soave-Wong-Sandler EoS model for a more accurate description of the pure component behavior. The pure component behavior and parameter requirements are described in RedlichKwong-Soave, and in Soave Alpha Functions.

\subsection{Schwarzentruber-Renon (SR-POLAR)}

The Schwartzentruber-Renon EoS is based on RedlichKwong EoS and can be used to model chemically nonideal systems with the same accuracy as activity coefficient property methods. This EoS is recommended for highly non-ideal systems at high temperatures and pressures, such as in methanol synthesis and supercritical extraction applications.

\section{Experimental data}

The experimental data used for working fluid EoS validation is reported in Table 1.

Table 1. Database for model validation.

\begin{tabular}{lllll}
\hline & $\begin{array}{l}\text { temperature } \\
\text { range } \\
(\mathrm{K})\end{array}$ & $\begin{array}{l}\text { pressure } \\
\text { range } \\
(\mathrm{MPa})\end{array}$ & $\begin{array}{l}\text { data } \\
\text { points }\end{array}$ & reference \\
property & $0-90$ & 284 & {$[8]-[10]$} \\
\hline $\begin{array}{l}\text { heat } \\
\text { capacity }\end{array}$ & $290-417$ & $0-90$ & 362 & {$[11]$} \\
\hline
\end{tabular}

The area of interest was pressure range of $7.39-20.00$ $\mathrm{MPa}$ and temperature range of $304-340 \mathrm{~K}$ just above fluid critical point (7.39 MPa, 304.25 K).

\section{Results and discussion}

Mean absolute percentage error (MAPE) was used to compare models quality by calculating differences between EoS and experimental data. Table 2 contains MAPE results for considered EoS. The Span-Wagner EoS results were added for reference as this EoS is considered to be the most accurate for supercritical $\mathrm{CO}_{2}$ applications [1]. Values in bold shows the most accurate equation for each property. 
Table 2. Database for model validation.

\begin{tabular}{lll}
\hline & $\begin{array}{l}\text { MAPE }\left(c_{\mathrm{p}}\right) \\
\text { EoS }\end{array}$ & $\begin{array}{l}\text { MAPE }(\rho) \\
(\%)\end{array}$ \\
\hline GERG'04 & $\mathbf{2 . 6 7 \%}$ & $0.29 \%$ \\
BWR-LS & $11.09 \%$ & $13.97 \%$ \\
CPA & $26.66 \%$ & $27.15 \%$ \\
PRWS & $14.49 \%$ & $8.60 \%$ \\
PRKS & $14.26 \%$ & $17.21 \%$ \\
RKS-BM & $12.84 \%$ & $15.36 \%$ \\
RKSWS & $14.26 \%$ & $17.21 \%$ \\
SR-POLAR & $12.84 \%$ & $15.36 \%$ \\
SW (reference) & $2.68 \%$ & $\mathbf{0 . 2 2 \%}$ \\
\hline
\end{tabular}

Results confirm that Helmoholtz-type EoS is the most accurate choice for supercritical $\mathrm{CO}_{2}$ applications. GERG'04 EoS shows almost the same accuracy as SW EoS. It was expected as GERG'04 EoS is based on SW EoS with minor modifications by Klimeck [13-14]. Virial-type as well cubic-type EoS accuracy is not acceptable and in most cases exceed $10 \%$.

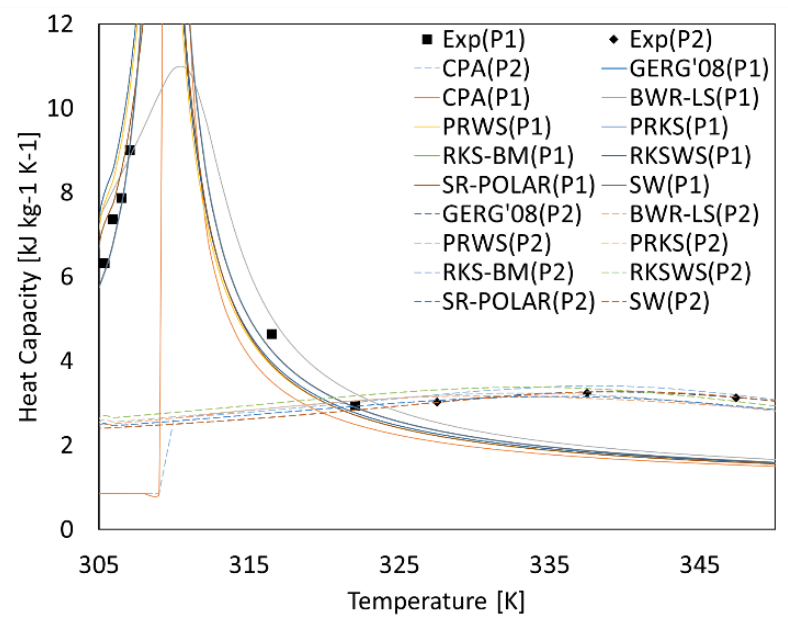

Fig. 3. Overview of different EoS results for isobaric heat capacity.

Graphical comparison between different EoS and experimental data is shown on Figure 3 and Figure 4. Two different isobars for each property were considered as follows: heat capacity $(\mathrm{P} 1=8.3 \mathrm{MPa}, \mathrm{P} 2=15.8$ $\mathrm{MPa})$, density $(\mathrm{P} 1=7.4 \mathrm{MPa}, \mathrm{P} 2=9 \mathrm{MPa})$. At lower pressures near the critical point peak in heat capacity can be observed.

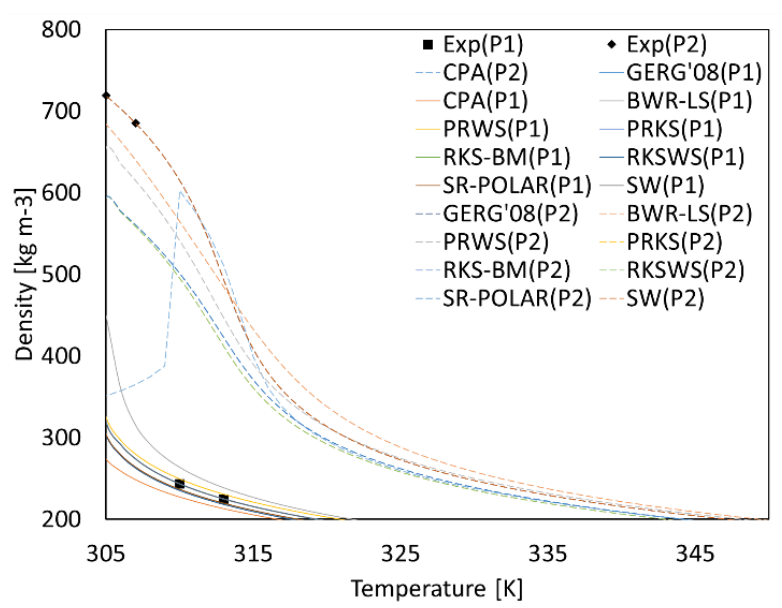

Fig. 4. Overview of different EoS results for density.

\section{Conclusions}

The paper investigates correctness of usage of different EoS in supercritical carbon dioxide applications. Eight different EoS available in popular commercial simulation software was tested. The area of interest selected for the study was pressure range of $7.39-20.00$ $\mathrm{MPa}$ and temperature range of $304-340 \mathrm{~K}$ just above fluid critical point $(7.39 \mathrm{MPa}, 304.25 \mathrm{~K})$ where the strongest deviation from ideal gas behavior can be observed. When considering pure component Helmoholtz-type EoS occurs to be the most accurate. The study confirms the statement by Zaho [1] that SW and GERG2008 should give similar results as both have the same theoretical foundations.

Based on the study it is recommended to use thermodynamic properties databases provided by The National Institute of Standards and Technology (NISTREFPROP) or Groupe Européen de Recherches Gazières (GERG) as they include accurate Helmoholtz-type EoS for carbon dioxide.

\section{Acknowledgements}

This scientific work was financially supported as a research project number $2015 / 19 / \mathrm{D} / \mathrm{ST} 8 / 02780$ by the National Science Centre, Poland.

\section{References}

1. Q. Zhao, M. Mecheri, T. Neveux, R. Privat, J.-N. Jaubert, Selection of a proper equation of state for the modeling of a supercritical $\mathrm{CO}_{2}$ brayton cycle: consequences on the process design, Ind. Eng. Chem. Res., 56, pp. 6841-6853 (2017)

2. Y. Chen, P. Lundqvist, P. Platell, Theoretical research of carbon dioxide power cycle application in automobile industry to reduce vehicle's fuel consumption, Applied Thermal Engineering, 25(1415), pp. $2041-2053$ (2005)

3. C. Oh, T. Lillo, W. Windes, T. Totemeier, R. Moore, Development of a supercritical carbon 
dioxide brayton cycle: Improving VHTR efficiency and testing material compatibility, INL/EXT-0601271 (2004)

4. M. Mazzoccoli, B. Bosio, E. Arato,, Analysis and comparison of equations-of-state with p-rho-t experimental data for $\mathrm{CO}_{2}$ and $\mathrm{CO}_{2}$-mixture pipeline transport, Energy Procedia 23, pp. $274-283$, The 6th Trondheim Conference on $\mathrm{CO}_{2}$ Capture, Transport and Storage (2012)

5. R. Span and W. Wagner, A new equation of state for carbon dioxide covering the fluid region from the triple point temperature to $1100 \mathrm{~K}$ at pressures up to 800MPa, J. Phys. Chem. Ref. Data 25(6), 1509$1596(1996)$

6. Z. Wang, B. Sun, and L. Yan, 2014, Improved density correlation for supercritical $\mathrm{CO}_{2}$, Chem. Eng. Tech. 38 (1) 75-84 (2015)

7. Y. Kim, C. Kim, and D. Favrat, Transcritical or supercritical $\mathrm{CO}_{2}$ cycles using both low- and hightemperature heat sources, Energy 43(1), pp. $402-$ 415 , 2nd International Meeting on Cleaner Combustion (2012)

8. L. B. Koppel, J. M. Smith, Thermal Properties of Carbon Dioxide in the Critical Region, J. Chem. Eng. Data, 5, pp. 437 (1960)

9. L. Dordian, J.-Y. Coxam, J. R. Quint, J.-P. E. Grolier, E. W. Lemmon, and S. G. Penoncello, Isobaric Heat Capacities of Carbon Dioxide and Argon Between 323 and $423 \mathrm{~K}$ and at Pressures up to $25 \mathrm{MPa}$, J. Supercrit. Fluids 8, pp. 228 (1995)

10. G. Ernst, G. Maurer, E. Wiederuh, Flow calorimeter for the accurate determination of the isobaric heat capacity at high pressures results for carbon dioxide, J. Chem. Thermodynamics, 21, pp. 53-65 (1989)

11. W. Duschek, R. Kleinrahm, W. Wagner, Measurement and correlation of the (pressure, density, temperature) relation of carbon dioxide, J. Chem. Thermodynamics, 32, pp. 827-840 (1990)

12. O. Kunz, W. Wagner, The GERG-2008 WideRange Equation of State for Natural Gases and Other Mixtures: An Expansion of GERG-2004, J. Chem. Eng. Data, 57 (11), pp. 3032-3091 (2012)

13. O. Kunz, R. Klimeck, W. Wagner, M. Jaeschke, The GERG-2004 Wide-Range Equation of State for Natural Gases and Other Mixtures, Düsseldorf (2007)

14. R. Klimeck, Entwicklung einer Fundamentalgleichung für Erdgase für das Gas- und Flüssigkeitsgebiet sowie das Phasengleichgewicht. Dissertation, Fakultät für Maschinenbau, RuhrUniversität Bochum, (2000)

15. M.R. Brulé, C.T. Lin, L.L. Lee, and K.E. Starling, J. AIChE, 28, pp. 616 (1982) 